\title{
ARTICLE
}

\section{Functional skull asymmetries in Carollia perspicillata (Phyllostomidae Gray, 1825: Carollinae)}

\author{
Pere M. Parés-Casanova*, Gerard Otin \\ Department of Animal Science, School of Agrifood and Forestry Science and Engineering (ETSEA), University of \\ Lleida, 25198 Lleida, Catalonia, Spain
}

\begin{abstract}
Analysing asymmetry in wild or domestic species enables the evaluation of the morphological responses to functional requirements and/or stress. This report is a study of the cranial asymmetry of Seba's short-tailed bat Carollia perspicillata by means of geometric morphometric techniques. We studied three types of bilateral asymmetries -fluctuating asymmetry, directional asymmetry and antisymmetry- using 15 skull landmarks on dorsal aspect of 45 skulls of Carollia perspicillata (21 males and 24 females) from different localities in Colombia. Directional asymmetry appeared to be significant and clearly higher than fluctuating asymmetry, with the braincase presenting the larger variance. There were no differences between sexes. Echolocation has a great anatomical effect on the bat cranium, and this would explain detected asymmetry. Acta Biol Szeged 64(1):37-42 (2020)
\end{abstract}

\author{
KEY WORDS \\ Chiroptera \\ echolocation \\ neurocranium \\ shape sexual dimorphism \\ Seba's short tailed bat \\ ARTICLE INFORMATION \\ Submitted \\ 01 June 2020. \\ Accepted \\ 24 July 2020. \\ *Corresponding author \\ E-mail: peremiquelp@ca.udl.cat
}

\section{Introduction}

Bilateral asymmetry refers to the non-correspondence in size and/or shape, and location of landmarks on opposite sides of the median sagittal plane. There are three main types of bilateral asymmetry: directional asymmetry (DA), fluctuating asymmetry (FA) and antisymmetry (AS) (Mendes 2008). DA is present when left and right sides consistently differ from each other (Klingenberg et al. 2002). FA is a pattern of subtle non-directional differences between sides (Klingenberg et al. 2002). AS, finally, represents a directional deviation from symmetry towards either the right or left side (Graham et al. 1993).

The use of FA variance has been considered a measure of developmental stability, constituting an indicator for the influence of environmental factors on a population (Tuyttens et al. 2005; Nuffel et al. 2007) and large values of FA are interpreted to reflect worse environmental conditions and hence decreased welfare (Tuyttens et al. 2005). DA and AS are considered inappropriate for the estimation of the underlying developmental instability because of their presumed heritable component (Graham et al. 1993; Tuyttens et al. 2005).

Carollia Gray, 1838 is a genus in the Phyllostomidae family, which is located on South and Central America, being among the most common mammal group in the
Neotropics (Gardner 2007; Bolzan et al. 2015). They are bats of robust and medium size, with prominent nose leaves, consisting of a lower part immediately surrounding the nostrils and a pronounced lancet extending dorsally, thought to be associated with sound emission for echolocation but trade-off with other sensory modalities such as olfaction and vision (Arbour et al. 2019). Some researchers have suggested that variation in nostrils size is the main source of the morphological plasticity of phyllostomes, although there is a lack of studies on the patterns of variation (López-Aguirre et al. 2015). Eight species are currently recognized (Gardner 2007): Carollia sowelli, C. subrufa, C. manu, C. monohernandezi, C. benkeithi, C. brevicauda, C. castanea and C. perspicillata (López-Aguirre et al. 2015). Colombia holds the highest variety of philostomide species, where until now four species have been described: C. brevicauda, C. castanea, C. monohernandezi and C. perspicillata (López-Aguirre et al. 2015). Seba's short-tailed bat (Carollia perspicillata) inhabits wet perennials and dry deciduous forests, usually below 1,000 meters of altitude, usually perched on groups of till 100 animals in caves, hollow trees, tunnels and sewers of roads. It feeds on fruits as well as pollen and insects (Brinkløv et al. 2011).

The skull is a complex morphological structure with high adaptative significance and often used in studies of asymmetries (Urošević et al. 2015). Variation in skull and 


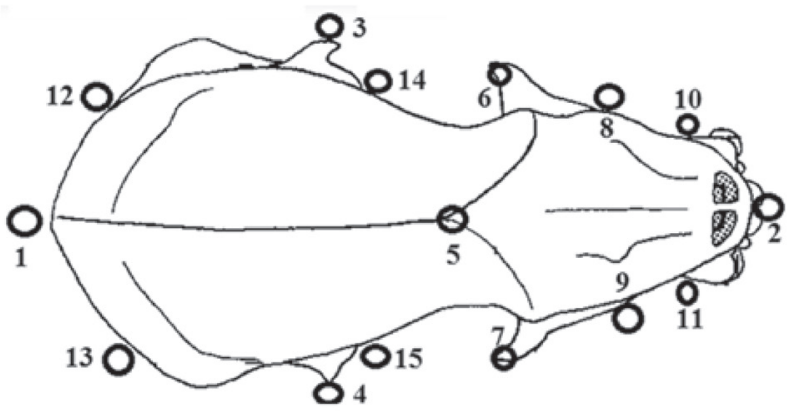

Figure 1. Carollia sp. Location of the 15 landmarks on the dorsal view.

body morphology has been used extensively to reconstruct ontogenetic processes in bats (McLellan 1984; Willig et al. 1986; Ruelas 2017; Ruelas and López 2018), but much less work has been done on asymmetries (López-Aguirre and Pérez-Torres 2015). Against this background, the main aims of our study were to (i) examine a sample of C. perspicillata for the presence of AS, FA and DA, (ii) test whether sexes differ in the extent of DA. We hypothesize that symmetry of skull in that species can be modified as an adaptation for echolocation.

\section{Materials and methods}

\section{Sample collection}

A total of 45 specimens of Carollia perspicillata (21 males and 24 females) originated from Colombia and initially collected for use in other studies was studied. This material is currently deposited in the Universidad del Cauca, in Popayán (Colombia). Damaged skulls had been previously excluded. Images were captured on the dorsal aspect using a digital camera (Nikon D1500) equipped with a lens (Nikon DX de 18-105 mm), with skull levelled on the horizontal plane. Each image included a $10 \mathrm{~mm}$ ruler. The images were later stored in JPEG format.

\section{Geometric morphometrics}

We employed landmark-based geometric morphometric techniques for the study. Fifteen landmarks -5 on the neurocranium -braincase, which surrounds and protects the brain-, 7 on the viscerocranium -which supports the functions of feeding and breathing, and forms the face in mammals- and 3 on the midline- (Fig. 1) were positioned on dorsal aspect of each skull using the TpsDig v. 2.26 software (Rohlf 2010). These landmarks, expressed as $x, y$ coordinates in Cartesian space, were considered to be homologous and topographically equivalent and sufficiently summarise the skull morphology of the dorsal structures. They were digitized by second author. Differences in size, position, translation, and rotation were deleted using Generalised Procrustes Analysis (Bookstein 1991). To quantify and minimize measurement error, skulls were digitized two times.

\section{Statistical analysis}

Prior to calculation of measurement error and asymmetry, the data were checked for AS. For this we used the Kolmogorov-Smirnov $D$ test to test for overall equal distribution of signed right-left hemiskull size, expressed as centroid size (CS, the square root of the sum of squared distance between each landmark and the skull centroid) (Bookstein 1991). A Procrustes ANOVA on Procrustes coordinates were used to assess the contribution of individual variation, DA ("Sides" as the fixed factor), FA ("Individuals*Sides" interaction), and measurement error to the overall shape variation (Klingenberg et al. 2002). Shape variation was separated into a symmetric component (among-individual variation) and an asymmetric component (within-individual variation), but only the asymmetric component was considered in this study. The asymmetric component was quantified through the landmark deviation of the original configuration from the symmetric consensus of the original and mirror images.

Shape symmetric differences between sexes were assessed by a Canonical Variates Analysis (CVA), with sex as the grouping variable using Mahalanobis distance and a 10000 permutations test. The pattern of asymmetric shape variation among the sexes was studied by means of a Principal Component Analysis (PCA). Furthermore, Partial Least Squares (PLS) analysis of covariation within the configuration of neurocranial and viscerocranial landmarks and a modularity check were performed to test if shape changes were independent between those two parts of the skull. Finally, we analysed allometry to determine whether size had an effect on shape asymmetry. Shape asymmetry (asymmetric component) was regressed onto CS (values log-transformed). The statistical significance of this regression was estimated using the permutation test with 10,000 iterations.

Morphometric and statistical analyses were conducted using the MorphoJ v. 1.07a (Klingenberg 2011) and PAST v. 2.17c (Hammer et al. 2001) packages.

\section{RESULTS}

\section{Measurement error and antisymmetry}

Kolmogorov-Smirnov test demonstrated that signed difference between right and left hemiskulls did not differ significantly $(D=0.088, p=0.844)$. Thus, we focused on the study of entire skull form FA and DA. We also visually 


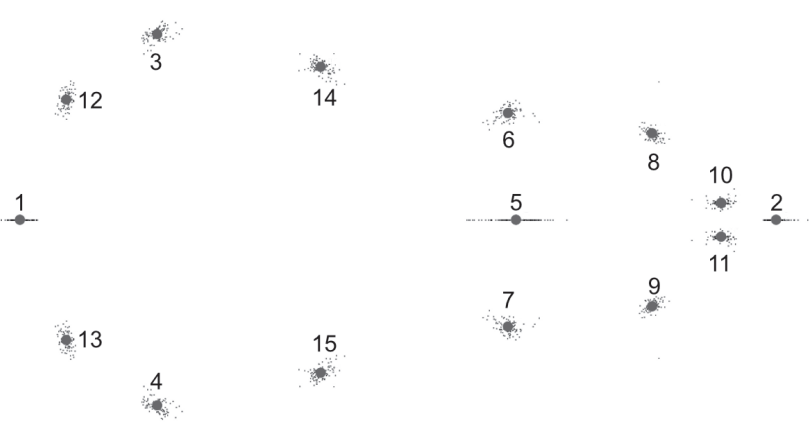

Figure 2. Scatter plot of points of left-right differences for each land mark after superimposition by the Procrustes algorithm. There was no evidence for clustering of these points that would have suggested antisymmetry.

Table 1. Loadings for Principal Components 1 and 2, which explained a $65.3 \%$ of the total observed variance (PC1 + PC2 $=39.1 \%+26.1 \%)$. Differences were located mainly on neurocranium length (coordinates $\times 3,4,12$ and 13) and orbital length and width (landmarks $x, y 14$ and 15) (in bold).

\begin{tabular}{lll}
\hline Coordinate & $\mathbf{P C 1}$ & $\mathbf{P C 2}$ \\
\hline x1 & 0 & 0 \\
y1 & 0.078497 & -0.47503 \\
x2 & 0 & 0 \\
y2 & -0.15667 & 0.225681 \\
x3 & -0.38106 & 0.150365 \\
y3 & 0.186871 & 0.271757 \\
x4 & $\mathbf{0 . 3 8 1 0 5 5}$ & -0.15037 \\
y4 & 0.186871 & 0.271757 \\
x5 & 0 & 0 \\
y5 & -0.2382 & -0.51175 \\
x6 & -0.12242 & 0.082166 \\
y6 & -0.10229 & -0.27036 \\
x7 & 0.122419 & -0.08217 \\
y7 & -0.10229 & -0.27036 \\
x8 & -0.11485 & 0.037143 \\
y8 & -0.00824 & 0.137144 \\
x9 & 0.114847 & -0.03714 \\
y9 & -0.00824 & 0.137144 \\
x10 & -0.02638 & -0.01853 \\
y10 & -0.14299 & 0.114636 \\
x11 & 0.026379 & 0.018531 \\
y11 & -0.14299 & 0.114636 \\
x12 & -0.21282 & 0.049994 \\
y12 & -0.06794 & 0.03923 \\
x13 & 0.212816 & -0.04999 \\
y13 & -0.06794 & 0.03923 \\
x14 & -0.28404 & 0.094742 \\
y14 & 0.292775 & 0.088144 \\
x15 & 0.284044 & -0.09474 \\
y15 & 0.292775 & 0.088144 \\
\hline
\end{tabular}

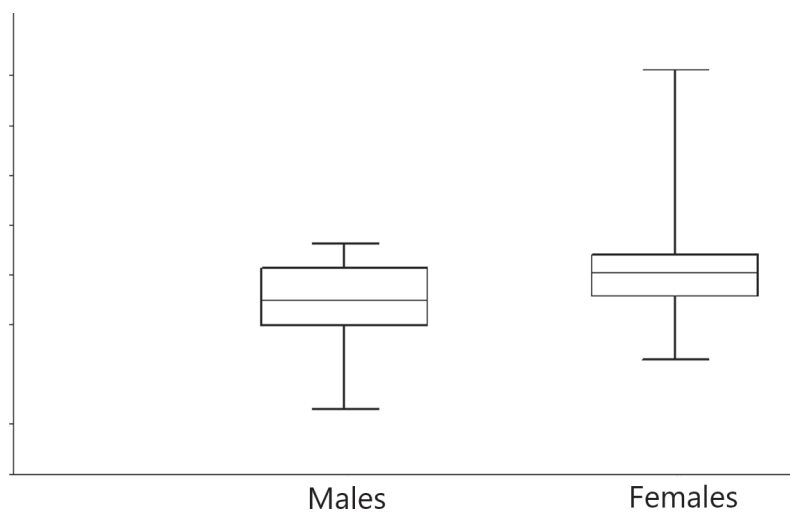

Figure 3. Box plot for skull sizes of C. perspicillata grouped by sex (21 males and 24 females), expressed as centroid size ( $Y$-axis). For each sample, the 25-75 percent quartiles are drawn using a box. The median is shown with a horizontal line inside the box. The minimal and maximal values are shown with short horizontal lines ("whiskers"). Skull sizes appeared statistically different for both sexes (Levene's value 0.677 , $F_{1,88}=7.959, p=0.0059$ ), being those of females clearly bigger.

examined scatter plot of points of left-right differences for each landmark after superimposition by the Procrustes algorithm. There was no evidence for clustering of these points (as the equivalent to bimodal distributions of leftright differences) that would have suggested AS (Fig. 2).

\section{Differences in size and shape asymmetry}

Skull sizes appeared statistically different for sexes (Levene's value $0.677, F_{1,88}=7.959, p=0.0059$ ), being those of females clearly bigger (Fig. 3), but there were no significant differences between females' and males' shape asymmetry (Mahalanobis distances $=0.839, p=0.262$ ), thus in subsequent analyses sexes were not analysed separately. Morphometric CVA space reflected a gamut of continuous variation and overlap for both sexes (Fig. 4).

Procrustes ANOVA showed that FA, DA, and individual

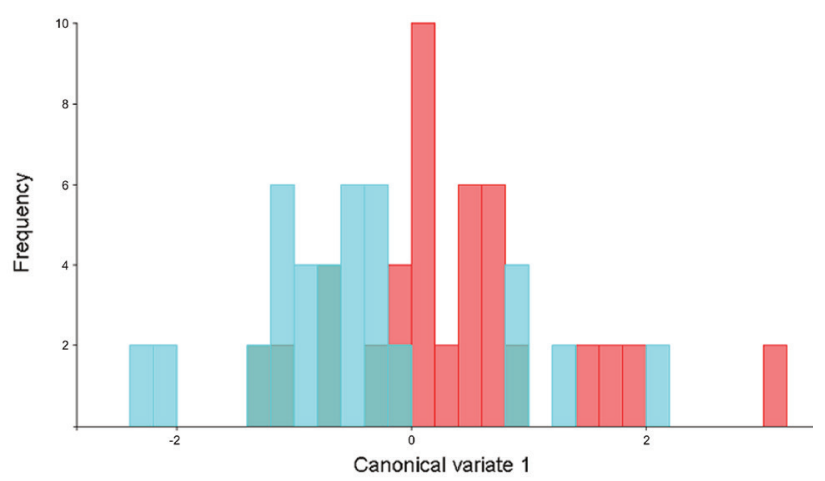

Figure 4. Canonical Variate Analysis results for the axis of CV1 for males (21) and females (24) of C. perspicillata. 


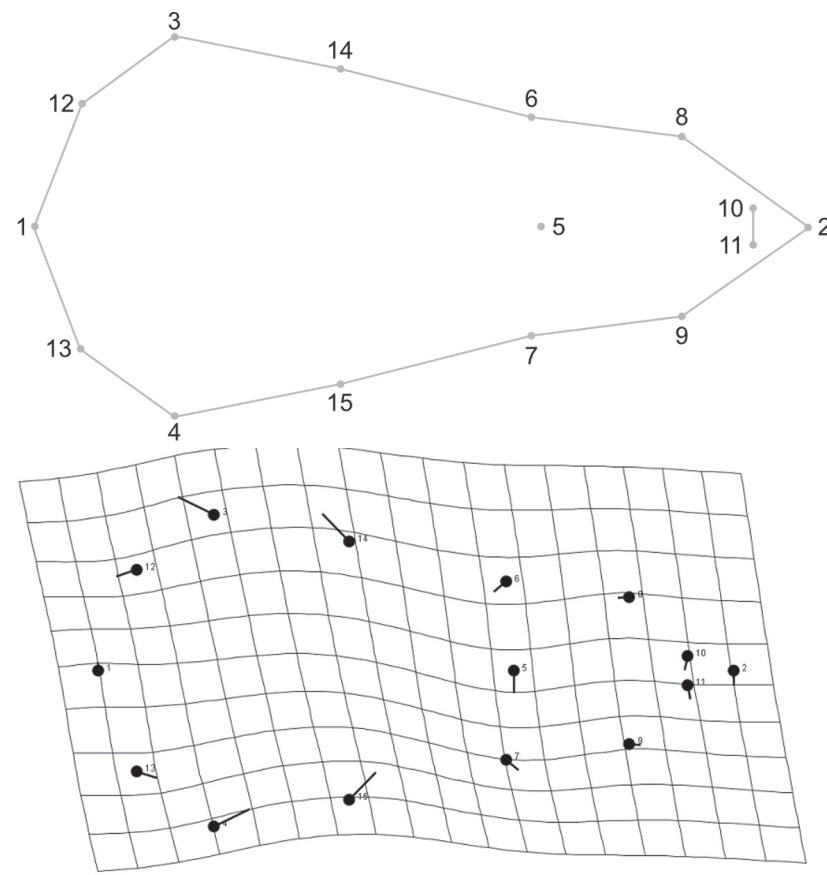

Figure 5. Deformation grid of shape differences detected by canonical variates for skulls of $C$. perspicillata $(n=45)$. Landmarks are represented by black filled circles. This grid produces a geometric description of shape to detect deformations relative to a general consensus to explain the shape change.

variation were found to exceed the error component, e.g., the contribution of measurement error to overall shape variation was small. FA and DA appeared statistically significant, but most of variation was concentrated in DA (89.5\%), contrasting with shape FA, which contributed only to $2.1 \%$ to total shape. Skull asymmetry varied primarily along two first PCs which explained a $65.3 \%$ of the total observed variance $(\mathrm{PC} 1+\mathrm{PC} 2=39.1 \%+$ 26.1\%). Shape changes detected by PC1 are illustrated on deformation grid (Fig. 5). Differences were located mainly on neurocranium length (coordinates $x 3,4,12$ and 13) and orbital length and width (landmarks $x, y 14$ and 15) (Table 1). These results prompted us to test whether the neurocranial area had a relatively independent shape change in viscerocranial variances.

The landmarks involving neurocranium $(1,3,4,12$, 13, 14 and 15) were selected as a block; all of the other landmarks composed a second block involving viscerocranium, which was our hypothesized partition (Fig. 7). PLS1 presented $66.1 \%$ of the total covariance and PLS2 presented $31.9 \%$, indicating that PLS1 represented the main covariance of two blocks. For PLS1, the pairwise correlation between blocks was $0.874(p<0.001)$, as noted in the plots distributed around the diagonal line of the PLS1 scores coordinate in Fig. 6. The $R V$ coefficient was

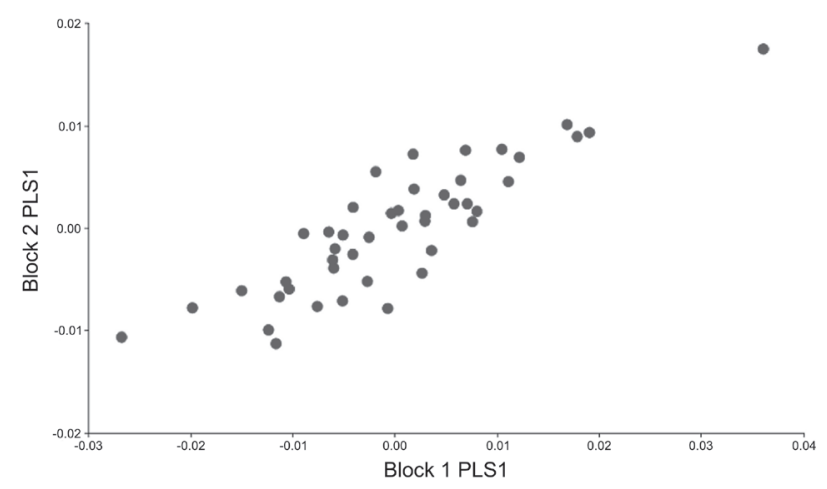

Figure 6. Scatter plot of the Partial Least Squares of two blocks (neurocranium and viscerocranium). PLS1 presented $66.1 \%$ of the total covariance, while PL2 presented 31.9\% (10 000 permutation test rounds).

0.472 , indicating that the overall strength of association between blocks was weak, although significant $(p<0.001)$. Based on the PLS analysis results, a modularity analysis was performed to evaluate whether both parts were separate modules. The $R V$ coefficient of the a priori hypothesis partition was 0.472 , which was similar to that of the PLS analysis. Moreover, the random partition result showed that there was no partition with an $R V$ less than or equal to the a priori hypothesis and thus, null hypothesis partition was accepted; the neurocranial and viscerocranial parts of the skull had different covariance. Therefore, the PLS analysis suggests that both parts of the C. perspicillata skull are separate modules, having no between-module integration.

\section{Associations of size and shape variation}

Correlations between the amount of shape asymmetry and CS were significant (3.2\% of shape $p=0.022)$.

\section{DISCUSSION}

The use of geometric morphometrics in this study gave rise to a specific exploration in skull asymmetry of $C$. perspicillata skull. Our results showed highly significant FA and DA in skull shape similar for both sexes, with a size -but not shape- sexual dimorphism.

FA has been used as an indicator of developmental stability in populations and it would imply a low capacity to respond to developmental accidents that occur under environmental and/or genetic conditions (Auffray et al. 1999). The contribution of shape FA to the total shape variation was significant for both sexes, but clearly lower than DA. This would be explained by the high level of canalisation of skull shape (Urošević et al. 2015), which would prevent large fluctuations so skull asymmetry, ex- 


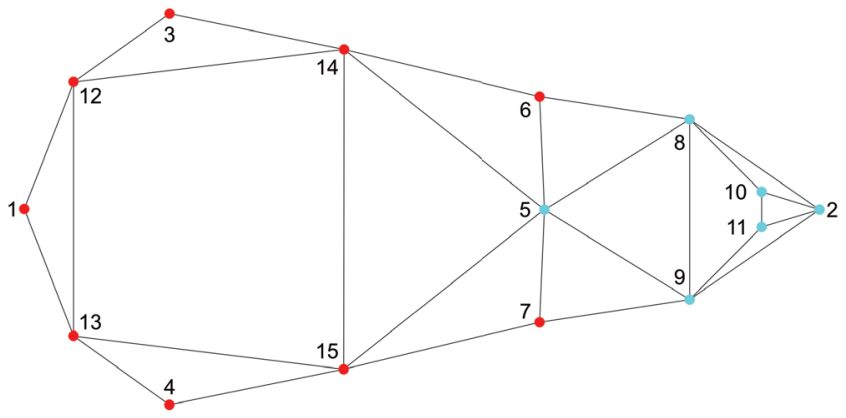

Figure 7. Modularity test results. The hypothesized partition: neurocranial part involving landmarks 1, 3 and 12-15, and the remaining landmarks as the visceral part; different colour presents different modules. Modules are units exhibiting a high degree of integration from many or strong interactions but relative independence from other such units.

pressed mainly as DA, must be interpreted as a reflection of adaptative traits.

Both PCA and CVA for asymmetric component showed that the braincase presented the larger variance. Echolocation has a great anatomical effect on the bat cranium (Brinkløv et al. 2011; Arbour et al. 2019). If we consider that this structural asymmetry may allow an asymmetric sound field and thus reduce left-right ambiguity in the echolocation (Au et al. 2006), the braincase, characterized by a significative DA, would reflect an asymmetrical support plate of the sensory apparatus (for instance ears) of the bats. To explain size sexual asymmetrical dimorphism, we argue that this can be due to that sexes have slight different in their preys, because different conformation of echolocation traits imply different ultrasound frequencies, and thus different prey sizes (Jakobsen et al. 2013). When allometry was tested we found that shape asymmetry and CS were correlated, meaning that an increase in size was accompanied by a more asymmetric shape. This presence of significant correlation of size and shape asymmetry suggests a developmental connection between them, meaning that size and shape are under similar developmental constraints, so the relative size of the skull has an effect on echolocation function in $C$. perspicillata.

The modularity test demonstrated that two skull modules -neurocranium and viscerocranium- would be units exhibiting a low degree of integration, e.g., with a relative high independence between them. In other words, neurocranium and viscerocranium would be distinct modules reflecting phenotypic and genetic variation.

\section{Acknowledgements}

We would like to thank to M.P. Rivas Pava for her generous support in handling the osteological material of the outstanding collection in Universidad del Cauca, in Popayán (Colombia). Moreover, we thank two anonymous reviewers whose helpful suggestions significantly improved the paper. We thank the Campus Iberus, Spain, for partial financial support for the field trips to museums.

\section{Availability of data and material}

The supplementary data for this article can be sent upon request to first author.

\section{References}

Arbour JH, Curtis AA, Santana SE (2019) Signatures of echolocation and dietary ecology in the adaptive evolution of skull shape in bats. Nat Commun 10:2036. https:// doi.org/10.1038/s41467-019-09951-y

Au WWL, Kastelein RA, Benoit-Bird KJ, Cranford TW, McKenna MF (2006) Acoustic radiation from the head of echolocating harbor porpoises (Phocoena phocoena). J Exp Biol 209(14):2726-2733.

Auffray JC, Debat V, Alibert P (1999) Shape asymmetry and developmental stability. In Chaplain MAJ, Singh GD, McLachlan JC, eds. On Growth and Form: Spatiotemporal Pattern Formation in Biology. Wiley, Chichester, pp 309-324.

Bolzan DP, Pessôa LM, Peracchi AL, Strauss RE (2015) Allometric patterns and evolution in neotropical nectarfeeding bats (Chiroptera, Phyllostomidae). Acta Chiropt 17(1):59-73.

Bookstein FL (1991) Morphometric tools for landmark data: geometry and biology. Cambridge University Press, Cambridge, 435 pp.

Brinkløv S, Jakobsen L, Ratcliffe JM, Kalko EKV, Surlykke A (2011) Echolocation call intensity and directionality in flying short-tailed fruit bats, Carollia perspicillata (Phyllostomidae). J Acoust Soc Am 129(1):427-435.

Gardner AL (2007) Mammals of South America. Volume 1. Marsupials, xenarthrans, shrews, and bats. The University of Chicago Press, Chicago. p. 690.

Graham JH, Freeman DC, Emlen JM (1993) Antisymmetry, directional asymmetry, and dynamic morphogenesis. Genetica, 89(1-3):121-137.

Hammer Ø, Harper DAT, Ryan PD (2001) PAST v. 2.17c. Palaeontol Electron 4(1):1-229.

Jakobsen L, Brinkløv S, Surlykke A (2013) Intensity and directionality of bat echolocation signals. Front Physiol 
4:89. doi: 10.3389/fphys.2013.00089.

Klingenberg CP (2011) MorphoJ: An integrated software package for geometric morphometrics. Mol Ecol Res 11(2):353-357.

Klingenberg CP, Barluenga M, Meyer A (2002) Shape analysis of symmetric structures: Quantifying variation among individuals and asymmetry. Evolution 56(10):1909-1920.

Klingenberg CP, McIntyre GS (1998) Geometric morphometrics of developmental instability: analyzing patterns of fluctuating asymmetry with procrustes methods. Evolution 52(5):1363-1375.

López-Aguirre C, Pérez-Torres J (2015) Asimetría cráneomandibular de Artibeus lituratus (chiroptera, phyllostomidae) en Colombia. Univ Sci 20(1):141-152.

López-Aguirre C, Pérez-Torres J, Wilson LAB (2015) Cranial and mandibular shape variation in the genus Carollia (Mammalia: Chiroptera) from Colombia: biogeographic patterns and morphological modularity. Peerj 3:e1197.

McLellan LJ (1984) A morphometric analysis of Carollia (Chiroptera, Phyllostomidae). Am Mus Novit 2791:1-35.

Mendes M (2008) Asymmetry measures and allometric growth parameter estimates for investigate effect of early feed restriction on deviation from bilateral symmetry in broiler chickens. Arch Tierzucht 6:611-619.

Nuffel AV, Tuyttens FAM, Dongen SV, Talloen W, Poucke EV, Sonck B, Lens L (2007) Fluctuating asymmetry in broiler chickens: a decision protocol for trait selection in seven measuring methods. Poult Sci 86:2555-2568.
Rohlf FJ (2010) tpsDig, digitize landmarks and outlines. Version 2.16. Stony Brook: Department of Ecology and Evolution, State University of New York at Stony Brook. Ruelas D (2017) Diferenciación morfológica de Carollia brevicauda y C. perspicillata (Chiroptera: Phyllostomidae) de Perú y Ecuador. Rev Peru Biol 24(4):363-382.

Ruelas D, López E (2018) Análisis morfogeométrico de las especies peruanas de Carollia (Chiroptera: Phyllostomidae). Mastozool Neotrop 25(2):419-438.

Tuyttens FAM, Maertens L, Van Poucke E, Van Nuffel A, Debeuckelaere S, Creve J, Lens L (2005) Measuring fluctuating asymmetry in fattening rabbits: a valid indicator of performance and housing quality? J Anim Sci 83(11):2645-2652.

Urošević A, Ljubisavljević K, Ivanović A (2015) Fluctuating asymmetry and individual variation in the skull shape of the common wall lizard (Podarcis muralis Laurenti, 1768) estimated by geometric morphometrics. Herpetol J 25(3):177-186.

Willig MR, Owen RD, Colbert RL (1986) Assessment of morphometric variation in natural populations: the inadequacy of the univariate approach. Syst Zool 35(2):195303. 